\title{
ESTADO DE CONOCIMIENTO DE LOS BRIOZOOS DULCEACUICOLAS DE CHILE
}

\section{CURRENT STATE OF KNOWLEDGE OF FRESHWATER BRYOZOA OF CHILE}

\author{
María Cristina Orellana Liebbe \\ Facultad de Ciencias, Universidad Católica de la Santísima Concepción. Casilla 297, Concepción, Chile. \\ Email:mcorella@ucsc.cl.
}

\begin{abstract}
RESUMEN
En Chile se han reportado seis especies de briozoos dulceacuícolas, pertenecientes a las familias Fredericellidae y Plumatellidae. La Familia Fredericellidae está representada sólo por la especie Fredericella sultana (Blumenbach 1779), y la Familia Plumatellidae por las especies: Plumatella punctata Hancock 1850, Plumatella patagonica Wiebach 1974, Plumatella casmiana Oka 1907, Plumatella repens (Linnaeus 1875) y Plumatella mukaii Wood 2001. El conocimiento sobre la taxonomía, ecología y biogeografías de las especies chilenas es todavía incipiente.
\end{abstract}

Palabras claves: Briozoos chilenos, taxonomía, Plumatellidae, Fredericellidae.

\section{ABSTRACT}

Six species of freshwater bryozoa have been reported in Chile, belonging to the families Fredericellidae and Plumatellidae. The Family Fredericellidae is only represented by the species Fredericella sultana (Blumenbach 1779), and the Family Plumatellidae for the species: Plumatella punctata Hancock 1850, Plumatella patagonica Wiebach 1974, Plumatella casmiana Oka 1907, Plumatella repens (Linnaeus 1875) and Plumatella mukaii Wood 2001. The knowledge on the taxonomy, ecology and biogeography of the Chilean species is still incipient.

KEYwords: Chilean bryozoa, taxonomy, Plumatellidae, Fredericellidae.

\section{INTRODUCCION}

El Phylum Bryozoa incluye tres clases, dos de las cuales, Staenolaemata y Gymnolaemata, son casi exclusivamente marinas, mientras que la tercera, la clase Phylactolaemata, incluye especies exclusivamente de agua dulce (Wood 1991). Si bien es reconocido por muchos autores (Ryland 1970, Wood 1991) que los briozoos de agua dulce son organismos comunes y abundantes, en todos los cuerpos de agua dulce alrededor del mundo (e.g. ríos, lagos, lagunas en áreas silvestres, como también en áreas inundadas y piletas ornamentales), el desarrollo del conocimiento de estos organismos en Chile y en Latinoamérica es muy escaso, y está lejos de ser una línea de investigación que haya perdurado en el tiempo (Moyano 1995,
Orellana 1999, Moyano et al.2003). En Chile el estudio de los briozoos de agua dulce comenzó en los primeros años del siglo XX, con el trabajo de Calvet (1904) en Tierra del Fuego. Este continuó, casi 70 años más tarde, con el trabajo de Wiebach (1974), quien describió briozoos de agua dulce de Puerto Natales. Dos décadas tomó para la siguiente referencia de este grupo; Valdovinos et al. (1996) menciona la presencia de estos organismos en la Región del BíoBío y Orellana (1999, 2004, datos no publicados), reanuda el estudio de estos organismos dando información acerca de la composición específica en varias lagunas de las regiones VII y VIII. Basado en este último estudio, Wood (2001) describió una nueva especie para Chile (Plumatella mukaii). Colateralmente a estos estudios, se ha generado tam- 
bién información orientada a la ecofisiología de algunas de las especies chilenas (Toledo 2004).

\section{CARACTERÍSTICAS GENERALES DE LOS PHYLACTOLAEMATA}

Los briozoos filactolemados están presentes tanto en aguas quietas como correntosas (Wood 1991), con temperaturas entre $0^{\circ}$ y $37^{\circ} \mathrm{C}$ (Marcus 1934 , Bushnell 1966, Shrivastava \& Rao 1985, Wood 1991), toleran un amplio rango de $\mathrm{pH}$ (Bushnell 1966, Tenney \& Woolacott 1966, Everitt 1975) y se desarrollan sobre una gama muy amplia de sustratos sumergidos (Cordiviola de Yuan 1977, Wood 1989). A veces se les encuentra creciendo conjuntamente con esponjas. Son organismos filtradores y viven por tanto, en aguas que contienen materia orgánica en suspensión y fitoplancton, considerándoseles como indicadores confiables del estado de las aguas donde ellos habitan (Marcus 1926 fide Banta \& Bakus 1991, Bushnell 1974, Sládecek 1980, Banta \& Bakus 1991).

Las colonias de briozoos filactolemados se caracterizan por poseer zooides tubulares quitinosos, con un epistoma y un lofóforo en forma de herradura, exceptuando las especies de la Familia Fredericellidae, cuyo lofóforo tiene forma circular. Los nuevos zooides se originan asexualmente por la replicación de polípidos mediante gemación. Se reproducen sexual y asexualmente aunque la reproducción sexual parece ser infrecuente (Bushnell 1966). La reproducción asexual en cambio, es el mecanismo dominante utilizado para dispersión y persistencia de un mismo genotipo a lo largo tiempo. Esta se lleva a cabo mediante un proceso de gemación interno que conduce a la formación de estatoblastos. Estos son de forma regular y bien definida, y son característicos solamente de la clase Phylactolaemata (Lacourt 1968). Se reconocen cuatro tipos de estatoblastos, considerando su morfología y función: a) sesoblastos o estatoblastos sésiles, que permanecen unidos al sustrato; b) flotoblastos, estatoblastos libres o flotadores (generalmente son más pequeños que los sesoblastos); c) leptoblastos, estatoblastos libres y d) piptoblastos, que corresponden a estatoblastos que permanecen al interior de la colonia, adhiriéndose a las paredes de ésta por medio de pequeñas proyecciones. El tipo, la morfología y tamaño de los estatoblastos constituyen elementos que son fundamentales para la sistemática de este grupo (Word 1989).

\section{Especies de filactolemados PRESENTES en Chile}

En la actualidad se reconocen seis especies para Chile, pertenecientes a las familias Fredericellidae y Plumatellidae. La Familia Fredericellidae se caracteriza porque las colonias son ramificadas, con zooides rastreros y en algunas ocasiones erectos, sin septos entre ellos. Los zooides son tubulares, largos y angostos, y pueden estar incrustados o parcialmente despegados del sustrato. Los zooides presentan un lofóforo circular compuesto por 20 a 24 tentáculos cortos. Los estatoblastos son del tipo piptoblastos, sin ornamentaciones. El largo de los piptoblastos varía entre 350 y $560 \mu \mathrm{m}$ y el ancho entre 170 y $300 \mu \mathrm{m}$ (Orellana 1999).

En Chile, esta familia está representada por una sola especie, Fredericella sultana (Blumenbach 1779) y se reconoce su presencia en zona de Tierra del Fuego (Calvet 1904), río Bío-Bío (Valdovinos et al. 1996) y laguna Pineda (36 $\left.50^{\prime} \mathrm{S} ; 7^{\circ} 56^{\prime} \mathrm{W}\right)$ en la VIII región y en el Ciénago del Name (3543'S; 72¹3'W) (VII Región) (Orellana 1999).

La familia Plumatellidae se caracteriza por presentar un lofóforo en forma de herradura compuesto por tentáculos de número variable (Tabla I), y más largos que los presentes en la familia Fredericellidae. Durante la historia de vida de la colonia en la mayoría de las especies se encuentran estatoblastos del tipo sesoblastos y flotoblastos y para unas pocas especies se encuentran además leptoblastos (Tabla I) (Lacourt 1968, Orellana 1999, Wood 2001, Toledo 2004).

En Chile esta Familia está representada por cinco especies. Plumatella punctata Hancock, 1850 ha sido descrita para Tierra del Fuego (Calvet 1904); Plumatella patagonica Wiebach 1974, registrada para Puerto Natales (Wiebach 1974), lagunas El Ciénago y Pineda (Orellana 1999). En tanto Plumatella casmiana y Plumatella repens están presentes en la laguna Lo Galindo (36 ${ }^{\circ} 48^{\prime}$ S; $\left.73^{\circ} 02^{\prime} \mathrm{W}\right)$ (Orellana 1999) y Laguna las Tres Pascualas (obs. personal). Plumatella mukaii está presente en Estación Villa Alegre ( $35^{\circ} 41^{\prime} \mathrm{S}$; $\left.71^{\circ} 41^{\prime} \mathrm{W}\right)$ y en todos los cuerpos de aguas de las regiones VII y VIII antes mencionados (Orellana 1999, Wood 2001). Estudios recientes realizados en la Laguna Verde de Hualpén (VIII Región) sugieren la presencia de una nueva especie de este género. 
Gayana 70(1), 2006

TABLA I. Número de tentáculos (rango) y dimensiones de los estatoblastos (rango) para algunas especies de la Familia Plumatellidae presentes en Chile. ${ }^{*}$ Sin datos.

TABLE I. Tentacle number (rank) and size of statoblasts (rank) for some species of the Family Plumatellidae present in Chile. * Without data.

\begin{tabular}{|c|c|c|c|c|c|c|c|}
\hline \multirow[b]{2}{*}{ Especie } & \multirow[b]{2}{*}{$\mathrm{N}^{\mathrm{o}}$ de tentáculos } & \multicolumn{2}{|c|}{ Sesoblasto $(\mu \mathrm{m})$} & \multicolumn{2}{|c|}{ Flotoblasto $(\mu \mathrm{m})$} & \multicolumn{2}{|c|}{ Leptoblasto $(\mu \mathrm{m})$} \\
\hline & & Largo & Ancho & Largo & Ancho & Largo & Ancho \\
\hline P. casmiana & $32-42$ & $*$ & * & $*$ & $*$ & $350-430$ & $190-260$ \\
\hline P. mukaii & $32-42$ & $325-580$ & $220-385$ & $310-490$ & $175-330$ & & \\
\hline P. repens & $40-60$ & $*$ & $*$ & $320-410$ & $215-300$ & & \\
\hline P. patagonica & $*$ & $*$ & $*$ & $550-750$ & $260-410$ & & \\
\hline
\end{tabular}

\section{REQUERIMIENTOS DE ESTUdIOS FUTUROS}

Las especies descritas para nuestro país son el resultado del estudio de una proporción muy pequeña de cuerpos de agua (algunos de la VII y VIII regiones), por lo cual se hace necesario un estudio sistemático, que abarque las distintas regiones del país, para contribuir al conocimiento de la biodiversidad de filactolemados. El conocimiento más acabado de este grupo puede ayudar a tomar decisiones mejor fundamentadas en cuanto a efectos de impacto ambiental en la biodiversidad de organismos de ambientes de agua dulce.

Por otra parte es necesaria una revisión del material recolectado para asegurar que las especies son válidas para el rango de distribución descrito, esto es especialmente necesario para $P$. punctata y $P$. patagonica, conocidas sólo para una o dos localidades en Chile.

El estudio de los en briozoos de agua dulce ha adquirido recientemente gran relevancia, ya que se ha demostrado que son hospedadores de microsporideos y mixosporideos (Okamura 1996, Okamura \& Wood 2002, Morris \& Adams 2002, Morris et al. 2002), organismos que causan una enfermedad que afecta el riñon de los peces (PKD), provocando grandes pérdidas en los cultivos de salmones en distintos lugares del mundo. Esta es una línea de investigación todavía inexplorada en nuestro país.

\section{AGRADECIMIENTOS}

Agradezco a J.M. Cancino por los comentarios y sugerencias a este trabajo. A Claudio Valdovinos por invitarme a participar del Taller de Limnología, y rescatar del anonimato a este grupo de organismos. Información de este trabajo parcialmente obtenida con el Proyecto DIN 06-2003.

\section{BIBLIOGRAFIA}

BANTA, W.C. \& B.T. BAKUs. 1991. Bryozoans as indicators of water quality in the Washington DC area. Final report to US Department of the Interior, District of Columbia Water Resources Research Center. 23 pp.

BushnelL, J.H. JR. 1966. Environmental relations of Michigan Ectoprocta, and dynamics of natural populations of Plumatella repens. Ecological Monographs 36: 95-123.

Bushnell, J.H. 1974. Bryozoa (Ectoprocta) En: Pollution ecology of freshwater invertebrates (Eds. C.W. Hart \& S.L.H. Fuller), pp. 157-194. Academic Press, New York

Calvet, L. 1904. Bryozoen. Ergebnisse der Hamburger Magalhaensischen Sammelreise 1892/93 3: 3940.

Cordiviola de Yuan, E. 1977. Endoprocta y Ectoprocta. En: Biota Acuática de Sudamérica Austral (Ed. Hulbert), pp 90-93. San Diego State University.

EveritT, B.C. 1975. Freshwater Ectoprocta: distribution and ecology of five species in southeastern Louisiana. Transactions of the American Microscopical Society 94: 130 - 134

LACOURT, A.W. 1968. A monograph of the freshwater Bryozoa - Phylactolaemata. Zoologische Verhandelingen 93: 1 - 159.

Marcus, E. 1934. Über Lophopus crystallinus (Pall.). Zoologische Jahrbücher Abteilung für Anatomie und Ontogenie der Tiere 58: 501 - 606.

Moyano, H. 1995. Bryozoa. En: Diversidad biológica de Chile (Eds. J.A. Simonetti, M.T.K. Arroyo, A.E. Spotorno \& E. Lozada), pp. 163-173. Comité Nacional de Diversidad Biológica, CONICYT, Santiago, Chile. 
Briozoos dulceacuícolas de Chile: Orellana, M.C.

Moyano, H., J.M. Cancino \& M.C. Orellana. 2003. Introduction to the 13th IBA International Conference. Chilean Bryozoology. Boletín de la Sociedad de Biología de Concepción, Chile 74: 23-34.

Morris J. \& A. AdAmS. 2002. Development of Schroedera plumatellae gen. n., sp. n. (Microsporidia) in Plumatella fungosa (Bryozoa: Phylactolaemata). Acta Protozoologica 41: 383-396.

Morris, J., D. Morris Ch. \& A. Adams. 2002. Development and release of malacosporean (Myxozoa) from Plumatella repens (Bryozoa: Phylactolaemata). Folia Parasitologica 49: 25-34.

OKAMURA, B. 1996. Occurrence, prevalence and the effects of the myxozoan Tetracapsula bryozoides parasitic in the freshwater bryozoan Cristatella mucedo (Bryozoa: Phylactolaemata). Folia Parasitologica 43: 262-266.

OkamuRa, B. \& T.S. Wood. 2002. Bryozoans as hosts for Tetracapsula bryosalmonae, the PKX organism. Journal of Fish Diseases 25: 469-475.

Orellana, M.C. 1999. Briozoos de agua dulce en Chile central (Bryozoa, Phylactolaemata). Tesis para optar al grado de Magister en Ciencias, mención Zoología, Universidad de Concepción. 67 pp.

Ryland, J. 1970. Bryozoans. Hutchinson University Library, London. $175 \mathrm{pp}$

Shrivastava, P. \& K.S. RaO. 1985. Ecology of Plumatella emarginata (Ectoprocta: Phylactolaemata) in the surface waters of Madhya Pradesh with note on its ocurrence in the protected waterworks of Bhopal (India). Environmental Pollution (Series A) 39: 123-130.
SLÁDECEK, V. 1980. Indicator value of freshwater Bryozoa Acta Hydrochimica and Hydrobiologica 8: 273-276.

Tenney, W.R. \& W.S. Woolacott. 1966. The occurrence and ecology of freshwater bryozoans in the headwaters of the Tennessee, Savannah, and Saluda River systems. Transaction of the American Microscopical Association 85: 241-245.

Toledo, B. 2004. Efecto de la concentración de oxígeno disuelto en el crecimiento colonial, en el tamaño de los zooides y número de tentáculos de Plumatella casmiana (Oka, 1907) (Bryozoa: Phylactolaemata). Informe Técnico, THB 500. Facultad de Ciencias, Universidad Católica de la Santísima Concepción. 21 pp.

Valdovinos, C., J. Stuardo \& J. Arenas. 1996. Estructura comunitaria del macrozoobentos de la zona de transición ritrón - potamón del río Bío-Bío. En: Evaluación de la calidad del agua y ecología del sistema limnético y fluvial del río Bío-Bío (Eds. F. Faranda \& O. Parra). Serie Monografías Científicas EULA, Universidad de Concepción 12: 217-248.

WIEBACH, F. 1974. Amazonische Moostiere III (Bryozoa). Amazoniana 5: 293-303.

Wood, T. S. 1991. Bryozoans. En: Ecology and classification of North American Freshwater invertebrates (Eds. J.H. Thorp \& A.P. Covich). Pp. 481-499. Academic Press, Inc. Portland.

Wood, T.S. 1989. Ectoproct bryozoans of Ohio. Ohio Biological Survey. Bulletin New Series 8(2): 1-70.

Wood, T.S. 2001. Plumatella mukaii, a new phylactolaemate bryozoan from Asia and South America. Hydrobiologia. 445: 51-56.

Fecha de recepción: 30.08 .05

Fecha de aceptación: 16.12.05 